AperTO - Archivio Istituzionale Open Access dell'Università di Torino

A multiplicity result for periodic solutions of second order differential equations with a singularity

This is the author's manuscript

Original Citation:

Availability:

This version is available http://hdl.handle.net/2318/151184

since 2015-12-02T14:07:11Z

Published version:

DOI:10.1016/j.na.2011.10.025

Terms of use:

Open Access

Anyone can freely access the full text of works made available as "Open Access". Works made available under a Creative Commons license can be used according to the terms and conditions of said license. Use of all other works requires consent of the right holder (author or publisher) if not exempted from copyright protection by the applicable law. 


\section{(3) \\ UNIVERSITÀ DEGLI STUDI DI TORINO}

This Accepted Author Manuscript (AAM) is copyrighted and published by Elsevier. It is posted here by agreement between Elsevier and the University of Turin. Changes resulting from the publishing process - such as editing, corrections, structural formatting, and other quality control mechanisms may not be reflected in this version of the text. The definitive version of the text was subsequently published in [Nonlinear Analysis: theory, methods and applications, 75, 12,2012,10.1016/j.na.2011.10.025].

You may download, copy and otherwise use the AAM for non-commercial purposes provided that your license is limited by the following restrictions:

(1) You may use this AAM for non-commercial purposes only under the terms of the CC-BY-NC-ND license.

(2) The integrity of the work and identification of the author, copyright owner, and publisher must be preserved in any copy.

(3) You must attribute this AAM in the following format: Creative Commons BY-NC-ND license (http://creativecommons.org/licenses/by-nc-nd/4.0/deed.en), [10.1016/j.na.2011.10.025 http://www.journals.elsevier.com/nonlinear-analysis-theory-methods-and-applications/] 


\title{
A MULTIPLICITY RESULT FOR PERIODIC SOLUTIONS OF SECOND ORDER DIFFERENTIAL EQUATIONS WITH A SINGULARITY
}

\author{
Alberto Boscaggin, Alessandro Fonda, Maurizio Garrione
}

\begin{abstract}
By the use of the Poincaré-Birkhoff fixed point theorem, we prove a multiplicity result for periodic solutions of a second order differential equation, where the nonlinearity exhibits a singularity of repulsive type at the origin and has linear growth at infinity. Our main theorem is related to previous results by Rebelo and Zanolin [15, 17, 18, 19, in connection with a problem raised by del Pino, Manásevich and Montero in 2.
\end{abstract}

AMS-Subject Classification. 34C25; 34B16, 37J10.

Keywords. Multiple periodic solutions; repulsive singularity; Poincaré-Birkhoff theorem.

\section{Introduction}

In [2], del Pino, Manásevich and Montero considered an equation like

$$
x^{\prime \prime}-\frac{1}{x^{\nu}}+\beta x=p(t),
$$

where $p: \mathbb{R} \rightarrow \mathbb{R}$ is continuous and $T$-periodic, $\nu \geq 1$, and $\beta>0$. They proved that, if

$$
\beta \neq\left(\frac{k \pi}{T}\right)^{2}, \quad \text { for every } k \in \mathbb{N},
$$

then there exists at least one $T$-periodic solution to 1.1 . In general, this condition is not eliminable. Indeed, if $\beta=\left(\frac{k \pi}{T}\right)^{2}$ for some positive integer $k$, some kind of resonance can occur: as shown in [1, Theorem 3], taking $p(t)=\epsilon \sin \left(\frac{2 \pi k}{T} t\right)$, with $|\epsilon|$ sufficiently small, no $T$-periodic solutions to (1.1) can exist.

Quoting the last sentence in [2],

"...the solution we are predicting in our "Fredholm alternative" for (1.1) is not necessarily unique, so the multiplicity problem for this simple equation is raised as an open question." 
In [15, 17, 18, 19], Rebelo and Zanolin analyzed the multiplicity problem assuming the forcing term to be of the form $p(t)=s+e(t)$, being $s$ a real parameter. By the use of the Poincaré-Birkhoff fixed point theorem, they proved that, for $|s|$ large enough, equation (1.1) may have a large number of $T$-periodic solutions. Their results apply to the wider class of $T$-periodic problems of the type

$$
\left\{\begin{array}{l}
x^{\prime \prime}+h(x)=s+e(t) \\
x(0)=x(T), x^{\prime}(0)=x^{\prime}(T),
\end{array}\right.
$$

where $h:] 0,+\infty[\rightarrow \mathbb{R}$ is a continuously differentiable function, with a suitable singularity of repulsive type at the origin, and linear growth at $+\infty$.

In this paper, similarly as in [7, 21], we consider the more general problem

$$
\left\{\begin{array}{l}
x^{\prime \prime}+g(t, x)=s w(t) \\
x(0)=x(T), x^{\prime}(0)=x^{\prime}(T),
\end{array}\right.
$$

where $g:[0, T] \times] 0,+\infty[\rightarrow \mathbb{R}$ satisfies some kind of Carathéodory conditions, with locally Lipschitz continuity in its second variable, and $w \in L^{\infty}(0, T)$. We will prove the following result.

Theorem 1.1. Assume that:

- there exist $\delta>0$ and a continuous function $f:] 0, \delta] \rightarrow \mathbb{R}$ such that

$$
g(t, x) \leq f(x), \quad \text { for a.e. } t \in[0, T] \text {, and every } x \in] 0, \delta],
$$

and

$$
\lim _{x \rightarrow 0^{+}} f(x)=-\infty, \quad \int_{0}^{\delta} f(x) d x=-\infty,
$$

- there exist a function $a \in L^{\infty}(0, T)$ and a positive integer $m$ such that

- uniformly for almost every $t \in[0, T]$,

$$
\lim _{x \rightarrow+\infty} \frac{g(t, x)}{x}=a(t)
$$

- for almost every $t \in[0, T]$,

$$
\left(\frac{m \pi}{T}\right)^{2}<a_{-} \leq a(t) \leq a_{+}<\left(\frac{(m+1) \pi}{T}\right)^{2},
$$

for suitable real constants $a_{-}, a_{+}$;

- the unique solution $\hat{x}(t)$ to

$$
\left\{\begin{array}{l}
x^{\prime \prime}+a(t) x=w(t) \\
x(0)=x(T), x^{\prime}(0)=x^{\prime}(T)
\end{array}\right.
$$

is strictly positive, i.e., $\hat{x}(t)>0$ for every $t \in[0, T]$. 
Then, there exists $s^{*}>0$ such that, for every $s \geq s^{*}$, problem (1.4 has at least

$$
\begin{cases}m+2 \text { solutions } & \text { if } m \text { is odd } \\ m+1 \text { solutions } & \text { if } m \text { is even. }\end{cases}
$$

Observe that $(1.6)$ is a nonresonance assumption with respect to the set

$$
\Sigma^{D}=\left\{\left(\frac{k \pi}{T}\right)^{2} \mid k=1,2, \ldots\right\},
$$

which is the spectrum of the differential operator $x \mapsto-x^{\prime \prime}$, with Dirichlet boundary conditions on $[0, T]$. This implies that we also have nonresonance with respect to the $T$-periodic problem, so that the Fredholm alternative ensures the uniqueness of the solution to (1.7).

Recall that, as shown in [7, Remark 6], condition (1.6) is not enough to ensure that the solution $\hat{x}(t)$ is positive; in the case when $w(t) \equiv 1$, some sufficient conditions (in term of some $L^{p}$-norm of $a(t)$ ) to guarantee this fact have been introduced in [20, Corollary 2.3].

We emphasize that, in comparison with the results obtained in [15, 17, 18, 19], besides the introduction of a possibly nonconstant function $w(t)$, we do not assume any differentiability hypothesis on the function $g(t, x)$, and the nonresonance assumption at $+\infty$ relies only on the asymptotic behavior of the quotient $g(t, x) / x$.

As it is clear, in the case $w(t) \equiv 1$ and $a(t) \equiv \beta \notin \Sigma^{D}$, with $\beta>0$, the unique solution to 1.7$)$ is strictly positive, being $\hat{x}(t) \equiv \frac{1}{\beta}$, so that, in the particular case of problem (1.3), we have the following.

Corollary 1.1. Assume that $h(x)$ is a locally Lipschitz continuous function such that

$$
\lim _{x \rightarrow 0^{+}} h(x)=-\infty, \quad \int_{0}^{1} h(x) d x=-\infty,
$$

and

$$
\lim _{x \rightarrow+\infty} \frac{h(x)}{x}=\beta,
$$

with $\beta>0$ satisfying 1.2 . Then, there exists $s^{*}>0$ such that, for every $s \geq s^{*}$, problem (1.3) has at least $N_{\beta}$ solutions, being

$$
N_{\beta}=2\left(\left\lfloor\frac{T \sqrt{\beta}}{\pi}\right\rfloor-\left\lfloor\frac{T \sqrt{\beta}}{2 \pi}\right\rfloor\right)+1 .
$$

(Here, for every positive number $a$, we denote by the symbol $\lfloor a\rfloor$ the greatest integer less than or equal to a.) 
Notice that

$$
\lim _{\beta \rightarrow+\infty} N_{\beta}=+\infty
$$

This fact could be related with a result in [9], where the case of superlinear growth at $+\infty$ was considered, as well as the case of a second repulsive singularity at a point $\bar{b}>0$. In both cases, the existence of infinitely many solutions was proved.

A result similar to Corollary 1.1 has been proved in [15, Theorem 2.5], where the function $h(x)$ was supposed to be continuously differentiable, and some conditions on $h^{\prime}(x)$ were assumed instead of (1.8).

The proof of Theorem 1.1 follows an argument introduced by del Pino, Manásevich and Murua in [3], in the context of asymmetric nonlinearities, motivated by a suspension bridge model proposed by Lazer and McKenna [13. In the same line, further generalizations were given in [7, 8, 21]. We first find a solution $\hat{x}_{s}(t)$ to 1.4 by means of topological degree arguments, and then, after a change of variable which transforms this solution into the origin, we use the Poincaré-Birkhoff fixed point theorem (see Section 2 for the details), to find the other solutions. In particular, for every integer

$$
k \in] \frac{T \sqrt{\beta}}{2 \pi}, \frac{T \sqrt{\beta}}{\pi}[,
$$

we find two solutions $x_{s, k}^{1}(t), x_{s, k}^{2}(t)$ such that $x_{s, k}^{i}(t)-\hat{x}_{s}(t)$ has exactly $2 k$ zeros in $[0, T[$, for $i=1,2$.

Let us now briefly summarize the content of the forthcoming sections. In Section 2. we recall the precise version of the Poincaré-Birkhoff theorem which we will use, as well as some useful preliminaries about the rotation number of plane paths. Section 3 is devoted to the proof of the main result. Finally, in Section 4 we give a more general statement which shows how condition (1.6) can be weakened, together with some final remarks.

\section{Preliminaries for the proof}

In this section, we introduce some preliminaries which will be useful for the proof of Theorem 1.1. First, we recall the notion of rotation number of a plane curve around the origin.

Definition 2.1. For $t_{1}<t_{2}$, let $z:\left[t_{1}, t_{2}\right] \rightarrow \mathbb{R}^{2}$ be an absolutely continuous path such that $z(t) \neq 0$ for every $t \in\left[t_{1}, t_{2}\right]$. The rotation number of $z(t)=(u(t), v(t))$ around the origin is defined as

$$
\operatorname{Rot}\left(z(t) ;\left[t_{1}, t_{2}\right]\right)=\frac{1}{2 \pi} \int_{t_{1}}^{t_{2}} \frac{v(t) u^{\prime}(t)-u(t) v^{\prime}(t)}{u(t)^{2}+v(t)^{2}} d t .
$$


It is well known that $\operatorname{Rot}\left(z(t) ;\left[t_{1}, t_{2}\right]\right)$ counts the normalized clockwise angular displacement of the curve $z(t)$ around the origin, in the time interval $\left[t_{1}, t_{2}\right]$. Precisely, writing $z(t)=\rho(t)(\cos \theta(t), \sin \theta(t))$, with $\rho(t), \theta(t)$ absolutely continuous functions, and $\rho(t)>0$, it holds that

$$
\operatorname{Rot}\left(z(t) ;\left[t_{1}, t_{2}\right]\right)=-\frac{\theta\left(t_{2}\right)-\theta\left(t_{1}\right)}{2 \pi} .
$$

In particular, when $z\left(t_{1}\right)=z\left(t_{2}\right)$, namely when $z(t)$ is a closed path, the number $\operatorname{Rot}\left(z(t) ;\left[t_{1}, t_{2}\right]\right)$ is an integer.

Remark 2.1. We will also need the following modified version of the rotation number, considered first in [6]. Precisely, for any positive real number $\kappa$ and any absolutely continuous path $z:\left[t_{1}, t_{2}\right] \rightarrow \mathbb{R}^{2}$ such that $z(t) \neq 0$ for every $t \in\left[t_{1}, t_{2}\right]$, we set

$$
\operatorname{Rot}_{\kappa}\left(z(t) ;\left[t_{1}, t_{2}\right]\right)=\frac{\kappa}{2 \pi} \int_{t_{1}}^{t_{2}} \frac{v(t) u^{\prime}(t)-u(t) v^{\prime}(t)}{\kappa^{2} u(t)^{2}+v(t)^{2}} d t .
$$

Such a definition corresponds to writing $z(t)=\rho(t)\left(\frac{1}{\kappa} \cos \theta(t), \sin \theta(t)\right)$ and, in general, gives a different value with respect to the classical rotation number of Definition 2.1. However, the remarkable fact (which is implicitly used in [6], see also [18, Theorem 4 and Remark 1] for a detailed proof) is that, for every integer $j$,

$$
\begin{aligned}
& \operatorname{Rot}\left(z(t) ;\left[t_{1}, t_{2}\right]\right)<j \Longleftrightarrow \operatorname{Rot}_{\kappa}\left(z(t) ;\left[t_{1}, t_{2}\right]\right)<j, \\
& \operatorname{Rot}\left(z(t) ;\left[t_{1}, t_{2}\right]\right)>j \Longleftrightarrow \operatorname{Rot}_{\kappa}\left(z(t) ;\left[t_{1}, t_{2}\right]\right)>j .
\end{aligned}
$$

We will need the following homotopy invariance of the rotation number.

Proposition 2.2. Let $z:\left[t_{1}, t_{2}\right] \times[0,1] \rightarrow \mathbb{R}^{2}$ be a continuous function, with $z(\cdot ; \lambda)$ absolutely continuous for every $\lambda \in[0,1]$. Assume that:

- $z(t ; \lambda) \neq 0$ for every $t \in\left[t_{1}, t_{2}\right]$ and every $\lambda \in[0,1]$;

- there exist $P, Q \in \mathbb{R}^{2} \backslash\{0\}$ such that, for every $\lambda \in[0,1]$,

$$
z\left(t_{1} ; \lambda\right)=P \text { and } z\left(t_{2} ; \lambda\right)=Q \text {. }
$$

Then

$$
\operatorname{Rot}\left(z(t ; 0) ;\left[t_{1}, t_{2}\right]\right)=\operatorname{Rot}\left(z(t ; 1) ;\left[t_{1}, t_{2}\right]\right) .
$$

This property follows from the fact that, defining the differential form

$$
\omega(u, v)=\frac{v d u-u d v}{u^{2}+v^{2}}
$$

it holds that

$$
\operatorname{Rot}\left(z(t ; \lambda) ;\left[t_{1}, t_{2}\right]\right)=\int_{z(\cdot ; \lambda)} \omega,
$$


for every $\lambda \in[0,1]$. Since $\omega$ is a closed differential form on $\mathbb{R}^{2} \backslash\{0\}$ and the paths $z(\cdot, 0)$ and $z(\cdot, 1)$ are joint by an admissible homotopy (with the same endpoints $P$ and $Q$ ), the conclusion follows from the standard theory of differential forms (see, for instance, [5, Chapter 2, Theorem 2]).

In the proof of the forthcoming Lemma 3.4, we will consider two paths $z_{1}, z_{2}$ : $[0, T] \rightarrow \mathbb{R}^{2}$ and a convex compact set $\mathcal{K}$ such that:

- $z_{1}(t) \notin \mathcal{K}$ for every $t \in[0, T]$

- $z_{2}(t)$ is a closed path such that $z_{2}(t) \in \mathcal{K}$ for every $t \in[0, T]$.

We will apply Proposition 2.2 with

$$
z(t ; \lambda)=z_{1}(t)-\left(\lambda z_{2}(t)+(1-\lambda) P\right),
$$

where $P=z_{2}(0)=z_{2}(T)$. In this situation, $z_{2}(t)$ is thus continuously deformed into its initial/final point $P$, so that it is possible to conclude that

$$
\operatorname{Rot}\left(z_{1}(t)-z_{2}(t) ;[0, T]\right)=\operatorname{Rot}\left(z_{1}(t)-P ;[0, T]\right) .
$$

Lastly, for the reader's convenience, we recall here the theorem which will be used in Section 3 to get our multiplicity result. We give the precise statement for the general case of a planar Hamiltonian system. In the following, we denote by

$$
J=\left(\begin{array}{cc}
0 & -1 \\
1 & 0
\end{array}\right)
$$

the standard symplectic matrix and by $\mathcal{D}(\Gamma)$ the open bounded region delimited by a Jordan curve $\Gamma \subset \mathbb{R}^{2}$ (according to the Jordan Theorem).

Theorem 2.3. Let $\mathcal{O}$ be a relatively open subset of $[0, T] \times \mathbb{R}^{2}$ and $H: \mathcal{O} \rightarrow$ $\mathbb{R}$ be such that $\nabla H(t, z)$ is a Carathéodory function (cf. [12]), locally Lipschitz continuous with respect to $z$. Assume further that

$$
[0, T] \times\{0\} \subset \mathcal{O} \quad \text { and } \quad \nabla H(t, 0) \equiv 0 .
$$

Finally, assume that there exist two Jordan curves $\Gamma_{0}, \Gamma_{\infty} \subset \mathbb{R}^{2}$, with

$$
0 \in \mathcal{D}\left(\Gamma_{0}\right) \subset \overline{\mathcal{D}\left(\Gamma_{0}\right)} \subset \mathcal{D}\left(\Gamma_{\infty}\right)
$$

both strictly star-shaped around the origin, and two positive integers $k_{0} \leq k_{\infty}$ such that: 
- for every $\bar{z} \in \overline{\mathcal{D}\left(\Gamma_{\infty}\right)}$, one has $(0, \bar{z}) \in \mathcal{O}$, and the (unique) solution to the Cauchy problem

$$
\left\{\begin{array}{l}
J z^{\prime}=\nabla H(t, z) \\
z(0)=\bar{z},
\end{array}\right.
$$

which we denote by $z(\cdot ; \bar{z})$, is defined on $[0, T]$, with $(t, z(t ; \bar{z})) \in \mathcal{O}$ for every $t \in[0, T]$

- $\operatorname{Rot}(z(t ; \bar{z}) ;[0, T])<k_{0}, \quad$ for every $\bar{z} \in \Gamma_{0}$;

- $\operatorname{Rot}(z(t ; \bar{z}) ;[0, T])>k_{\infty}, \quad$ for every $\bar{z} \in \Gamma_{\infty}$.

Then, for every integer $k \in\left[k_{0}, k_{\infty}\right]$, the problem

$$
\left\{\begin{array}{l}
J z^{\prime}=\nabla H(t, z) \\
z(0)=z(T)
\end{array}\right.
$$

has at least two (distinct) solutions $z_{1, k}(t), z_{2, k}(t)$, with

$$
z_{1, k}(0), z_{2, k}(0) \in \overline{\mathcal{D}\left(\Gamma_{\infty}\right)} \backslash \mathcal{D}\left(\Gamma_{0}\right)
$$

such that

$$
\operatorname{Rot}\left(z_{1, k}(t) ;[0, T]\right)=\operatorname{Rot}\left(z_{2, k}(t) ;[0, T]\right)=k .
$$

Observe that the local Lipschitz continuity assumption on $\nabla H(t, z)$ ensures the uniqueness for every Cauchy problem associated with the equation in (2.1). In particular, since $\nabla H(t, 0) \equiv 0$, it turns out that $z(t ; \bar{z}) \neq 0$ for every $t \in[0, T]$, provided that $\bar{z} \neq 0$. As a consequence, the rotation numbers appearing in the statement are well defined.

Theorem 2.3 is actually a consequence of the Poincaré-Birkhoff fixed point theorem, in the version by W.Y. Ding [4], when applied to the Poincaré map associated with the planar system $J z^{\prime}=\nabla H(t, z)$, as an area preserving map

$$
\Psi: \overline{\mathcal{D}\left(\Gamma_{\infty}\right)} \rightarrow \mathbb{R}^{2}, \quad \bar{z} \mapsto z(T ; \bar{z})
$$

The result in [4], however, requires an extra assumption, i.e. the strictly starshapedness of the outer boundary of the annular region, as recently pointed out in [14]. For a proof of Theorem 2.3 under this stronger assumption, we refer to [16. Corollaries 2 and 3]. See also [10] for a recent account on the state of the art concerning the Poincaré-Birkhoff theorem.

Theorem 2.3 will be applied to an equation of the type

$$
u^{\prime \prime}+h(t, u)=0
$$


with $h(t, u)$ defined on a relatively open subset $\Omega \subset[0, T] \times \mathbb{R}$ and such that $h(t, 0) \equiv 0$; such an equation will be obtained starting from the differential equation in (1.4) by means of a suitable change of variable. Indeed, (2.3) is equivalent to the planar Hamiltonian system $J z^{\prime}=\nabla H(t, z)$, with the position $z=(u, v)$, $\mathcal{O}=\Omega \times \mathbb{R} \subset[0, T] \times \mathbb{R}^{2}$, and, for $(t, u, v) \in \mathcal{O}$,

$$
H(t, u, v)=\frac{1}{2} v^{2}+\int_{0}^{u} h(t, \xi) d \xi .
$$

In the situation of equation 2.3 , the solutions $z(t)$ to the equivalent Hamiltonian system 2.1 having rotation number equal to $k$ correspond to $T$-periodic solutions $u(t)$ with $2 k$ zeros in $[0, T[$.

\section{Proof of Theorem 1.1}

Let us first clarify our regularity assumptions. The function $g:[0, T] \times] 0,+\infty[\rightarrow \mathbb{R}$ is supposed to be an $L^{\infty}$-Carathéodory function which is locally Lipschitz continuous in its second variable, that is:

- $g(\cdot, x) \in L^{\infty}(0, T)$, for every $x>0$,

- for every compact interval $I \subset] 0,+\infty\left[\right.$, there exists a constant $C_{I}>0$ such that, for almost every $t \in[0, T]$ and for every $x, y \in I$,

$$
|g(t, x)-g(t, y)| \leq C_{I}|x-y|
$$

For further convenience, we set

$$
\mathcal{N}(x, y)=\sqrt{\frac{1}{x^{2}}+x^{2}+y^{2}}, \quad x>0, y \in \mathbb{R} .
$$

Such a function plays the role of a "norm" in the phase-plane for solutions of equations with a singularity at the origin, in the sense that a solution $x(t)$ is considered "large" when $\mathcal{N}\left(x(t), x^{\prime}(t)\right)$ is "large". In particular, this is the case when $x(t)^{2}+x^{\prime}(t)^{2}$ is large, or when $x(t)$ approaches the origin.

Our proof of Theorem 1.1 is based on four preliminary lemmas. The first one concerns the global continuability for the Cauchy problems associated with the differential equation in 1.4 .

Lemma 3.1. For every $s \in \mathbb{R}$, the unique solution to the Cauchy problem

$$
\left\{\begin{array}{l}
x^{\prime \prime}+g(t, x)=s w(t) \\
x(0)=\bar{x}>0 \\
x^{\prime}(0)=\bar{y}
\end{array}\right.
$$

is globally defined on $[0, T]$. 
Proof. Let us suppose by contradiction that there exists a solution $x(t)$ of (3.1) whose maximal interval of definition is $[0, \tau$ [ for $\tau<T$. By standard arguments in the theory of initial value problems, one has

$$
\limsup _{\sigma \rightarrow \tau^{-}} \mathcal{N}\left(x(\sigma), x^{\prime}(\sigma)\right)=+\infty \text {. }
$$

A contradiction will then be achieved by using some properties of the rotation number of large solutions which have been proved in [11. Indeed, by the arguments therein, it has to be

$$
\lim _{\sigma \rightarrow \tau^{-}} \operatorname{Rot}\left(\left(x(t)-1, x^{\prime}(t)\right) ;[0, \sigma]\right)=+\infty .
$$

On the other hand, following the computations in [11, Lemma 2], it is possible to see that the time needed for large solutions to perform a complete rotation around $(1,0)$ is bounded below by a positive constant, so that the solution necessarily has to perform only a finite number of rotations in the time interval $[0, \tau[$. We thus have a contradiction with 3.2 .

In the second lemma, by topological degree arguments (developed in [21]), we find a first solution of (1.4), for $s>0$ sufficiently large.

Lemma 3.2. There exist $s_{1}>0$ and two positive constants $0<c<C$ such that, for every $s \geq s_{1}$, problem (1.4) has a solution $\hat{x}_{s}(t)$ satisfying

$$
c \leq \frac{\hat{x}_{s}(t)}{s} \leq C, \quad \text { for every } t \in[0, T]
$$

In particular,

$$
\lim _{s \rightarrow+\infty} \hat{x}_{s}(t)=+\infty, \quad \text { uniformly in } t \in[0, T] .
$$

Proof. Let us define, for $t \in[0, T]$ and $x \in \mathbb{R}$, the truncated function

$$
\widetilde{g}(t, x)=\left\{\begin{array}{lll}
g(t, x) & \text { if } & x \geq 1 \\
g(t, 1) & \text { if } & x \leq 1
\end{array}\right.
$$

Since, uniformly for almost every $t \in[0, T]$,

$$
\lim _{x \rightarrow+\infty} \frac{\widetilde{g}(t, x)}{x}=a(t),
$$

and the unique solution of $(1.7)$ is positive, using [21, Theorem 2.1] we have that, for every $s$ large enough, there exists a solution $\hat{x}_{s}(t)$ of

$$
\left\{\begin{array}{l}
x^{\prime \prime}+\widetilde{g}(t, x)=s w(t) \\
x(0)=x(T), x^{\prime}(0)=x^{\prime}(T) .
\end{array}\right.
$$


In particular, from the proof of [21, Theorem 2.1] it also follows that (3.3) is satisfied for suitable constants $c, C>0$. Indeed, such a solution is proved to be of the form

$$
\hat{x}_{s}(t)=s\left(\hat{x}(t)+\epsilon_{s}(t)\right),
$$

with $\hat{x}(t)$ as in Theorem 1.1, and $\left\|\epsilon_{s}\right\|_{\infty} \leq \frac{1}{2} \min _{t \in[0, T]} \hat{x}(t)$. Hence, 3.3 is satisfied for

$$
c=\frac{1}{2} \min _{t \in[0, T]} \hat{x}(t) \quad \text { and } \quad C=\max _{t \in[0, T]} \hat{x}(t)+\frac{1}{2} \min _{t \in[0, T]} \hat{x}(t) .
$$

Clearly, (3.3) implies that $x_{s}(t) \rightarrow+\infty$ uniformly in $t \in[0, T]$, so that, for large values of $s, \hat{x}_{s}(t) \geq 1$ for every $t \in[0, T]$, and hence $\hat{x}_{s}(t)$ solves (1.4).

We now perform the following change of variable:

$$
u(t)=\frac{x(t)-\hat{x}_{s}(t)}{s} .
$$

In this way, the solution $\hat{x}_{s}(t)$ is transformed into the origin, and also rescaled by a factor $s$, as suggested by formula (3.3). Accordingly, the differential equation in (1.4) is changed into

$$
u^{\prime \prime}+h_{s}(t, u)=0,
$$

where we have set, for simplicity,

$$
h_{s}(t, u)=\frac{g\left(t, s u+\hat{x}_{s}(t)\right)-g\left(t, \hat{x}_{s}(t)\right)}{s} .
$$

Notice that equation 3.5 is now of the type considered in the discussion after Theorem 2.3. that is to say, $h_{s}(t, u)$ is an $L^{\infty}$-Carathéodory function, locally Lipschitz continuous in $u$, which is well defined on a relatively open subset $\Omega_{s}$ of $[0, T] \times \mathbb{R}$, namely

$$
\Omega_{s}=\left\{(t, u) \in[0, T] \times \mathbb{R} \mid u>-\frac{\hat{x}_{s}(t)}{s}\right\},
$$

and $h_{s}(t, 0) \equiv 0$. Observe in particular that, for the Cauchy problem

$$
\left\{\begin{array}{l}
u^{\prime \prime}+h_{s}(t, u)=0, \\
u(0)=\bar{u}>-\hat{x}_{s}(0) / s, \\
u^{\prime}(0)=\bar{v},
\end{array}\right.
$$

there is global continuability on $[0, T]$. Indeed, $u(t)$ is a solution if and only if $x(t)=s u(t)+\hat{x}_{s}(t)$ solves 3.1 with $x(0)>0$. By Lemma 3.1, $x(t)$ is globally extendable on $[0, T]$, so that the same holds for $u(t)$.

Henceforth, we set

$$
\mathcal{D}_{s}=\left\{(u, v) \in \mathbb{R}^{2} \mid u>-\frac{\hat{x}_{s}(0)}{s}\right\} .
$$

In view of the previous discussion, the Poincaré operator 2.2 associated with the planar system equivalent to 3.5 is well defined on $\mathcal{D}_{s}$. 
The next lemma deals with the construction of the inner Jordan curve $\Gamma_{0}$ of Theorem 2.3, which, as a matter of fact, will be taken as a circumference around the origin. Such a construction is possible, provided that the parameter $s$ is large enough.

Lemma 3.3. There exist $\tilde{r} \in] 0, c / 2\left[\right.$, with $c$ as in (3.3), and $s_{2} \geq s_{1}$ such that, for every $s \geq s_{2}$,

- $\overline{\mathcal{B}}(0, \tilde{r}) \subset \mathcal{D}_{s}$, where $\overline{\mathcal{B}}(0, \tilde{r})$ denotes the closed ball of radius $\tilde{r}$ centered at the origin;

- for every $u:[0, T] \rightarrow \mathbb{R}$, solution to 3.5 satisfying $u(0)^{2}+u^{\prime}(0)^{2}=\tilde{r}^{2}$, it holds that

$$
\operatorname{Rot}\left(\left(u(t), u^{\prime}(t)\right) ;[0, T]\right)< \begin{cases}\frac{m+1}{2} & \text { if } m \text { is odd } \\ \frac{m}{2}+1 & \text { if } m \text { is even. }\end{cases}
$$

Proof. We begin with the following claims.

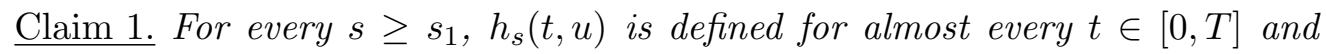
every $u \in[-c / 2, c / 2]$, where $c$ is as in (3.3); moreover, it holds that

$$
\lim _{s \rightarrow+\infty}\left(h_{s}(t, u)-a(t) u\right)=0,
$$

uniformly for almost every $t \in[0, T]$ and every $u \in[-c / 2, c / 2]$.

Proof of Claim 1. From the definition, we see that $h_{s}(t, u)$ is well defined for

$$
u>-\frac{\hat{x}_{s}(t)}{s},
$$

so that, by (3.3), the first part of the claim is proved.

Computing now the expression in (3.6) and using (3.3), we have, for $u \in[-c / 2, c / 2]$,

$$
\begin{aligned}
\left|h_{s}(t, u)-a(t) u\right| \leq & \left|\frac{g\left(t, s u+\hat{x}_{s}(t)\right)-a(t)\left(s u+\hat{x}_{s}(t)\right)}{s}\right|+\left|\frac{a(t) \hat{x}_{s}(t)-g\left(t, \hat{x}_{s}(t)\right)}{s}\right| \\
= & \left(u+\frac{\hat{x}_{s}(t)}{s}\right)\left|\frac{g\left(t, s u+\hat{x}_{s}(t)\right)-a(t)\left(s u+\hat{x}_{s}(t)\right)}{s u+\hat{x}_{s}(t)}\right|+ \\
& +\frac{\hat{x}_{s}(t)}{s}\left|\frac{a(t) \hat{x}_{s}(t)-g\left(t, \hat{x}_{s}(t)\right)}{\hat{x}_{s}(t)}\right| \\
\leq & (c+C)\left|\frac{g\left(t, s u+\hat{x}_{s}(t)\right)-a(t)\left(s u+\hat{x}_{s}(t)\right)}{s u+\hat{x}_{s}(t)}\right|+ \\
& +C\left|\frac{a(t) \hat{x}_{s}(t)-g\left(t, \hat{x}_{s}(t)\right)}{\hat{x}_{s}(t)}\right| .
\end{aligned}
$$

The conclusion is thus achieved, in view of 1.5$)$, since $\hat{x}_{s}(t) \rightarrow+\infty$ and $s u+\hat{x}_{s}(t) \rightarrow$ $+\infty$, uniformly in $t \in[0, T]$. 
Claim 2. There exist $b, \widetilde{r}$, with $0<b<\widetilde{r}<c / 2$ and $\widetilde{s}_{1} \geq s_{1}$ such that, for every $s \geq \widetilde{s}_{1}$, one has

- $\overline{\mathcal{B}}(0, \widetilde{r}) \subset \mathcal{D}_{s}$,

- for every $u:[0, T] \rightarrow \mathbb{R}$, solution to (3.5) satisfying $u(0)^{2}+u^{\prime}(0)^{2}=\widetilde{r}^{2}$, it holds that

$$
b^{2} \leq u(t)^{2}+u^{\prime}(t)^{2} \leq\left(\frac{c}{2}\right)^{2}
$$

for every $t \in[0, T]$.

Proof of Claim 2. Set $\rho(t)=\sqrt{u(t)^{2}+u^{\prime}(t)^{2}}$ and fix

$$
\widetilde{r}=\frac{c}{8} \exp \left(-\frac{1+a_{+}}{2} T\right),
$$

where $a_{+}$is as in (1.6). Observe that, since $\widetilde{r}<c / 2$, we have $\overline{\mathcal{B}}(0, \widetilde{r}) \subset \mathcal{D}_{s}$. We begin to prove the second inequality in (3.7), namely that $\rho(t)<c / 2$ for every $t \in[0, T]$. Notice that $\rho(0)=\widetilde{r}<c / 2$.

Assume by contradiction that there exists $\bar{t} \in[0, T]$ such that

$$
\rho(t)<\frac{c}{2} \text { for every } t \in\left[0, \bar{t}\left[, \quad \text { and } \quad \rho(\bar{t})=\frac{c}{2} .\right.\right.
$$

From equation 3.5 , we get

$$
\rho^{\prime}(t)=\frac{u^{\prime}(t)\left(u(t)-h_{s}(t, u(t))\right)}{\sqrt{u(t)^{2}+u^{\prime}(t)^{2}}} .
$$

In view of Claim 1 , since $|u(t)| \leq \rho(t) \leq c / 2$ for every $t \in[0, \bar{t}]$, there exists $\widetilde{s}_{1} \geq s_{1}$ such that, for every $s \geq \widetilde{s}_{1}$ and almost every $t \in[0, T]$,

$$
\left|h_{s}(t, u(t))-a(t) u(t)\right| \leq \frac{\widetilde{r}}{T} .
$$

Hence, by elementary inequalities, we achieve, from (3.8),

$$
\rho^{\prime}(t) \leq \frac{1+a_{+}}{2} \rho(t)+\frac{\widetilde{r}}{T}
$$

By Gronwall's lemma, we get

$$
\rho(\bar{t}) \leq\left(\rho(0)+\frac{\widetilde{r} \bar{t}}{T}\right) \exp \left(\frac{1+a_{+}}{2} \bar{t}\right) \leq 2 \widetilde{r} \exp \left(\frac{1+a_{+}}{2} T\right)=\frac{c}{4},
$$

whence the contradiction.

The proof of the other inequality in (3.7), i.e., $\rho(t)>b$ for every $t \in[0, T]$, is similar. Indeed, it suffices to exploit a time inversion argument, by observing that the function $\widetilde{u}(\sigma)=u(T-\sigma)$ satisfies the equation $\widetilde{u}^{\prime \prime}(\sigma)+h_{s}(T-\sigma, \widetilde{u}(\sigma))=0$. 
Hence, Gronwall's lemma can be used just as before, and the conclusion follows by choosing

$$
b=\frac{\widetilde{r}}{4} \exp \left(-\frac{1+a_{+}}{2} T\right)
$$

Going back to the proof of Lemma 3.3, recall that, from assumption (1.6),

$$
\frac{T \sqrt{a_{+}}}{2 \pi}<\frac{m+1}{2}
$$

hence, it is possible to fix $\zeta>0$ so small that

$$
\frac{T \sqrt{a_{+}}}{2 \pi}\left(1+\frac{c \zeta}{2 b^{2} \min \left\{a_{+}, 1\right\}}\right)<\frac{m+1}{2} .
$$

Moreover, in view of Claim 1 , there exists $s_{2} \geq \widetilde{s}_{1}$ such that, for $s \geq s_{2}$, it holds that

$$
\left|h_{s}(t, u)-a(t) u\right| \leq \zeta
$$

for almost every $t \in[0, T]$ and every $u \in[-c / 2, c / 2]$. Let now $u:[0, T] \rightarrow \mathbb{R}$ be a solution to (3.5), satisfying $u(0)^{2}+u^{\prime}(0)^{2}=\widetilde{r}^{2}$. In view of Claim 2 , we have $b^{2} \leq u(t)^{2}+u^{\prime}(t)^{2} \leq(c / 2)^{2}$ for every $t \in[0, T]$, so that

$$
\begin{aligned}
\operatorname{Rot}_{\sqrt{a_{+}}}\left(\left(u(t), u^{\prime}(t)\right) ;[0, T]\right)= & \frac{\sqrt{a_{+}}}{2 \pi} \int_{0}^{T} \frac{u^{\prime}(t)^{2}+h_{s}(t, u(t)) u(t)}{u^{\prime}(t)^{2}+a_{+} u(t)^{2}} d t \\
\leq & \frac{\sqrt{a_{+}}}{2 \pi}\left(\int_{0}^{T} \frac{u^{\prime}(t)^{2}+a(t) u(t)^{2}}{u^{\prime}(t)^{2}+a_{+} u(t)^{2}} d t+\right. \\
& \left.\quad+\int_{0}^{T} \frac{\left(h_{s}(t, u(t))-a(t) u(t)\right) u(t)}{u^{\prime}(t)^{2}+a_{+} u(t)^{2}} d t\right) \\
\leq & \frac{T \sqrt{a_{+}}}{2 \pi}\left(1+\frac{c \zeta}{2 b^{2} \min \left\{a_{+}, 1\right\}}\right) \\
< & \frac{m+1}{2} \leq \begin{cases}\frac{m+1}{2} & \text { if } m \text { is odd } \\
\frac{m}{2}+1 & \text { if } m \text { is even } .\end{cases}
\end{aligned}
$$

By the property recalled in Remark 2.1, we conclude.

The last lemma concerns the construction of the outer Jordan curve $\Gamma_{\infty}$ appearing in Theorem 2.3, which will turn out to be a translation of a level curve of the function $\mathcal{N}(x, y)$. Notice that, now, we do not need to enlarge $s$ any more.

Lemma 3.4. For every $s \geq s_{2}$, there exists a strictly star-shaped Jordan curve $\Upsilon_{s}$ around the origin such that 
- denoting by $\mathcal{D}\left(\Upsilon_{s}\right)$ the open bounded region delimited by $\Upsilon_{s}$, one has

$$
\overline{\mathcal{B}}(0, \widetilde{r}) \subset \mathcal{D}\left(\Upsilon_{s}\right) \subset \overline{\mathcal{D}\left(\Upsilon_{s}\right)} \subset \mathcal{D}_{s}
$$

where $\widetilde{r}$ is as in Lemma 3.3 ;

- for every $u:[0, T] \rightarrow \mathbb{R}$, solution to 3.5 satisfying $\left(u(0), u^{\prime}(0)\right) \in \Upsilon_{s}$, it holds that

$$
\operatorname{Rot}\left(\left(u(t), u^{\prime}(t)\right) ;[0, T]\right)>m \text {. }
$$

Proof. Fix $s \geq s_{2}$. Since $\widetilde{r}<c / 2$, in view of 3.3 we can fix $\hat{r}$ such that

$$
\widetilde{r}<\hat{r}<\frac{\hat{x}_{s}(0)}{s} .
$$

We prove the following claims.

Claim 1. There exists $R_{s}>0$ such that, if

$$
(u, v) \in \mathcal{D}_{s} \quad \text { and } \quad \mathcal{N}\left(s u+\hat{x}_{s}(0), s v+\hat{x}_{s}^{\prime}(0)\right) \geq R_{s}
$$

then

$$
\sqrt{u^{2}+v^{2}} \geq \hat{r}
$$

Proof of Claim 1. If $u \leq-\hat{r}$, the inequality clearly holds. On the other hand, if $u>-\hat{r}$, writing explicitly the expression of $\mathcal{N}\left(s u+\hat{x}_{s}(0), s v+\hat{x}_{s}^{\prime}(0)\right)$ and using elementary inequalities, we have, in view of 3.9 ,

$$
\begin{aligned}
2 s^{2}\left(u^{2}+v^{2}\right) & \geq R_{s}^{2}-2\left(\hat{x}_{s}(0)^{2}+\hat{x}_{s}^{\prime}(0)^{2}\right)-\frac{1}{\left(s u+\hat{x}_{s}(0)\right)^{2}} \\
& >R_{s}^{2}-2\left(\hat{x}_{s}(0)^{2}+\hat{x}_{s}^{\prime}(0)^{2}\right)-\frac{1}{\left(-s \hat{r}+\hat{x}_{s}(0)\right)^{2}},
\end{aligned}
$$

so that we conclude choosing $R_{s}$ large enough.

Claim 2. There exists $\hat{R}_{s} \geq R_{s}$ such that, for every $x:[0, T] \rightarrow \mathbb{R}$, solution to the differential equation in (1.4) satisfying

$$
\mathcal{N}\left(x(t), x^{\prime}(t)\right) \geq \hat{R}_{s} \text { for every } t \in[0, T],
$$

it holds that

$$
\operatorname{Rot}\left(\left(x(t)-\hat{x}_{s}(0), x^{\prime}(t)-\hat{x}_{s}^{\prime}(0)\right) ;[0, T]\right)>m .
$$

Proof of Claim 2. First of all, fix $\alpha \in](m \pi / T)^{2}, a_{-}[$and, accordingly, thanks to 1.5 and (1.6), choose $d>\hat{x}_{s}(0)$ such that

$$
\alpha\left(x-\hat{x}_{s}(0)\right) \leq g(t, x)-s w(t), \quad \text { for a.e. } t \in[0, T], \text { and every } x \geq d .
$$


Secondly, fix $\eta>0$ so small that

$$
\frac{\pi}{\sqrt{\alpha}}+4 \eta<\frac{T}{m}
$$

Let $\hat{R}_{s} \geq R_{s}$ be such that $\mathcal{N}\left(\hat{x}_{s}(0), \hat{x}_{s}^{\prime}(0)\right)<\hat{R}_{s}$. Let $x:[0, T] \rightarrow \mathbb{R}$ be a solution to the differential equation in (1.4) satisfying $\mathcal{N}\left(x(t), x^{\prime}(t)\right) \geq \hat{R}_{s}$ for every $t \in[0, T]$. In the following of the proof, we will possibly enlarge $\hat{R}_{s}$, taking care of the fact that all the estimates will be independent of the solution considered.

Writing $\left(x(t)-\hat{x}_{s}(0), x^{\prime}(t)-\hat{x}_{s}^{\prime}(0)\right)$ in polar coordinates for $t \in[0, T]$, namely

$$
x(t)=\hat{x}_{s}(0)+\rho(t) \cos \theta(t), \quad x^{\prime}(t)=\hat{x}_{s}^{\prime}(0)+\rho(t) \sin \theta(t),
$$

a standard computation yields, for every $t \in[0, T]$,

$$
-\theta^{\prime}(t)=\frac{x^{\prime}(t)\left(x^{\prime}(t)-\hat{x}_{s}^{\prime}(0)\right)+(g(t, x(t))-s w(t))\left(x(t)-\hat{x}_{s}(0)\right)}{\left(x(t)-\hat{x}_{s}(0)\right)^{2}+\left(x^{\prime}(t)-\hat{x}_{s}^{\prime}(0)\right)^{2}} .
$$

We are going to show that the time needed for $\left(x(t), x^{\prime}(t)\right)$ to perform a whole revolution around the point $\left(\hat{x}_{s}(0), \hat{x}_{s}^{\prime}(0)\right)$ is strictly less than $T / m$.

We first consider the case when $x(0)>d$ and $m=1$.

Step 1. We claim that, enlarging $\hat{R}_{s}$, if necessary, there is a first time instant $t_{1} \in$

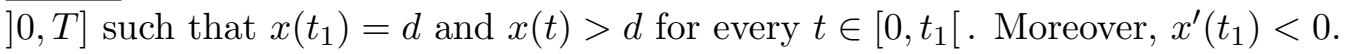

To this aim, we first show that, up to choosing $\hat{R}_{s}$ larger if necessary, we have

$$
-\theta^{\prime}(t) \geq \sin ^{2}(\theta(t))+\alpha \cos ^{2}(\theta(t))-\frac{\eta}{2 T} \min \{\alpha, 1\},
$$

whenever $x(t) \geq d$. Indeed, by $(3.12)$ and $(3.10)$, one has, for $x(t) \geq d$,

$$
-\theta^{\prime}(t) \geq \sin ^{2}(\theta(t))+\alpha \cos ^{2}(\theta(t))+\frac{\hat{x}_{s}^{\prime}(0) x^{\prime}(t)-\hat{x}_{s}^{\prime}(0)^{2}}{\left(x(t)-\hat{x}_{s}(0)\right)^{2}+\left(x^{\prime}(t)-\hat{x}_{s}^{\prime}(0)\right)^{2}}
$$

and elementary arguments show that, if $\hat{R}_{s}$ is large,

$$
\left\{\begin{array}{l}
\mathcal{N}(x, y) \geq \hat{R}_{s} \\
x \geq d,
\end{array} \Longrightarrow\left|\frac{\hat{x}_{s}^{\prime}(0) y-\hat{x}_{s}^{\prime}(0)^{2}}{\left(x-\hat{x}_{s}(0)\right)^{2}+\left(y-\hat{x}_{s}^{\prime}(0)\right)^{2}}\right| \leq \frac{\eta}{2 T} \min \{\alpha, 1\} .\right.
$$

Suppose by contradiction that $x(t)>d$ for every $t \in[0, T]$. From (3.13), we get

$$
\frac{\pi}{\sqrt{\alpha}} \geq\left.\frac{1}{\sqrt{\alpha}} \arctan \left(\frac{1}{\sqrt{\alpha}} \tan \theta(t)\right)\right|_{0} ^{T}=\int_{\theta(T)}^{\theta(0)} \frac{d \theta}{\sin ^{2} \theta+\alpha \cos ^{2} \theta} \geq T\left(1-\frac{\eta}{2 T}\right),
$$

which contradicts (3.11). Notice that this argument also shows that

$$
0<t_{1}<\frac{1}{\sqrt{\alpha}}\left(\arctan \left(\frac{1}{\sqrt{\alpha}} \tan \theta(0)\right)-\arctan \left(\frac{1}{\sqrt{\alpha}} \tan \theta\left(t_{1}\right)\right)\right)+\eta .
$$

Moreover, since $x^{\prime \prime}(t)<0$ for every $t \in\left[0, t_{1}\right]$, it follows that $x^{\prime}\left(t_{1}\right)<0$. 
Step 2. Choosing $\hat{R}_{s}$ large enough, one has

$$
-\theta^{\prime}(t)>0, \quad \text { for a.e. } t \in[0, T] .
$$

Indeed, if $x(t) \geq d$, this follows from the computations made in Step 1. On the other hand, if $x(t) \in] 0, d\left[\right.$, since $\mathcal{N}\left(x(t), x^{\prime}(t)\right)$ is large for every $t \in[0, T]$, either $x(t)$ is near the singularity or $\left|x^{\prime}(t)\right|$ is large. Formula $(3.16)$ then follows from the fact that $\lim _{x \rightarrow 0^{+}}(g(t, x)-s w(t))=-\infty$ uniformly for almost every $t \in[0, T]$, similarly as in [11, Lemma 2].

Arguing as in [11, Lemma 2] again, up to enlarging $\hat{R}_{s}$ we can find a second time instant $t_{2}>t_{1}$, with

$$
t_{2}-t_{1}<\eta
$$

such that $\left.x\left(t_{2}\right)=d, x^{\prime}\left(t_{2}\right)>0, x(t) \in\right] 0, d[$ for every $t \in] t_{1}, t_{2}[$, and $\pi<\theta(0)-$ $\theta\left(t_{2}\right)<2 \pi$.

Step 3. We claim that there exists $\left.\left.t_{3} \in\right] t_{2}, T\right]$ such that $\theta(0)-\theta\left(t_{3}\right)=2 \pi$.

Assume the contrary, that is, $\theta(0)-\theta(t)<2 \pi$ for every $t \in\left[t_{2}, T\right]$. By convexity reasons it has to be $x(t)>d$ in a right neighborhood of $t_{2}$.

If $x(t)>d$ for every $\left.t \in] t_{2}, T\right]$, with the same computations as in (3.14), together with 3.15$)$, we get

$$
t_{1}+\left(T-t_{2}\right)<\frac{\pi}{\sqrt{\alpha}}+2 \eta,
$$

a contradiction with (3.11), in view of (3.17). Therefore, there exists $\left.\left.t^{\prime} \in\right] t_{2}, T\right]$ such that $x\left(t^{\prime}\right)=d$, with $x(t)>d$ for $\left.t \in\right] t_{2}, t^{\prime}[$ (see Figure 1). Then, as before,

$$
\begin{aligned}
t_{1}+\left(t^{\prime}-t_{2}\right)< & \frac{1}{\sqrt{\alpha}}\left(\arctan \left(\frac{1}{\sqrt{\alpha}} \tan \theta\left(t_{2}\right)\right)-\arctan \left(\frac{1}{\sqrt{\alpha}} \tan \theta\left(t^{\prime}\right)\right)+\right. \\
& \left.+\arctan \left(\frac{1}{\sqrt{\alpha}} \tan \theta(0)\right)-\arctan \left(\frac{1}{\sqrt{\alpha}} \tan \theta\left(t_{1}\right)\right)\right)+2 \eta \\
< & \frac{\pi}{\sqrt{\alpha}}+2 \eta .
\end{aligned}
$$

Hence, using (3.17) and (3.11), we see that $t^{\prime}<T-\eta$. Consequently, the computations in [11, Lemma 2] imply that there exists $\left.t^{\prime \prime} \in\right] t^{\prime}, t^{\prime}+\eta\left[\right.$ such that $x\left(t^{\prime \prime}\right)=\hat{x}_{s}(0)$ and $x^{\prime}\left(t^{\prime \prime}\right)<\hat{x}_{s}^{\prime}(0)$, so that $\theta(0)-\theta\left(t^{\prime \prime}\right)>2 \pi$, a contradiction.

From the above discussion, we can also conclude that

$$
t_{3}-t_{2}<\frac{1}{\sqrt{\alpha}}\left(\arctan \left(\frac{1}{\sqrt{\alpha}} \tan \theta\left(t_{2}\right)\right)-\arctan \left(\frac{1}{\sqrt{\alpha}} \tan \theta\left(t_{3}\right)\right)\right)+2 \eta .
$$

Step 4. We have just proved that, in the phase-plane, $\left(x(t), x^{\prime}(t)\right)$ performs at least one turn around the point $\left(\hat{x}_{s}(0), \hat{x}_{s}^{\prime}(0)\right)$ in the time from 0 to $T$. In particular, we have the following upper bound for the time needed to perform such a revolution:

$$
t_{3}=t_{1}+\left(t_{2}-t_{1}\right)+\left(t_{3}-t_{2}\right) \leq \frac{\pi}{\sqrt{\alpha}}+4 \eta<\frac{T}{m} .
$$




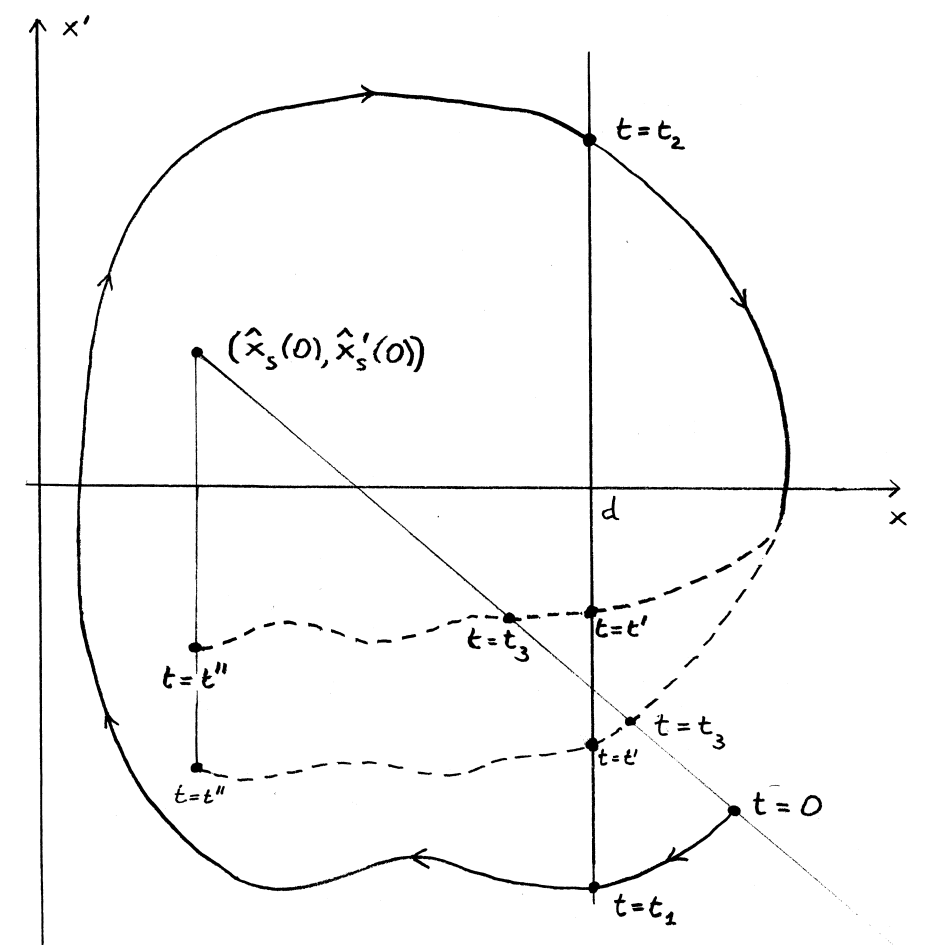

Figure 1: Two possible behaviors for the solution in the phase-plane.

In view of (3.16), the proof of Claim 2 is completed in the case when $x(0)>d$ and $m=1$. It can be easily seen that analogous considerations permit to conclude also in the case when $x(0) \leq d$.

If $m>1$, we can argue as above for any of the subsequent revolutions, with the same upper bounds on the time needed for each of them, until $m$ turns are performed. Hence, the time needed to perform $m$ revolutions has to be strictly less than $T$, and we conclude the proof of Claim 2 in view of (3.16).

We are now ready to conclude the proof of Lemma 3.4. Let $\mathcal{K}_{s}$ be a suitable closed rectangle in the half-plane $\{x>0\}$ such that $\left(\hat{x}_{s}(t), \hat{x}_{s}^{\prime}(t)\right) \in \mathcal{K}_{s}$ for every $t \in[0, T]$. Up to enlarging $\hat{R}_{s}$, it is not restrictive to assume that

$$
\mathcal{N}(x, y) \geq \hat{R}_{s} \quad \Longrightarrow \quad(x, y) \notin \mathcal{K}_{s} .
$$

As a standard consequence of the global continuability (the elastic property, cf. [18, Lemma 10]), there exists $\widetilde{R}_{s} \geq \hat{R}_{s}$ such that, for any $x:[0, T] \rightarrow \mathbb{R}$, solution to the differential equation in (1.4), one has

$$
\mathcal{N}\left(x(0), x^{\prime}(0)\right) \geq \widetilde{R}_{s} \quad \Longrightarrow \quad \mathcal{N}\left(x(t), x^{\prime}(t)\right) \geq \hat{R}_{s} \quad \text { for every } t \in[0, T] .
$$

Set now

$$
\Upsilon_{s}=\left\{(u, v) \in \mathcal{D}_{s} \mid \mathcal{N}\left(s u+\hat{x}_{s}(0), s v+\hat{x}_{s}^{\prime}(0)\right)=\widetilde{R}_{s}\right\}
$$


By Claim $1, \overline{\mathcal{B}}(0, \widetilde{r}) \subset \mathcal{D}\left(\Upsilon_{s}\right)$; moreover, $\Upsilon_{s}$ is a strictly star-shaped Jordan curve around the origin. Let $u:[0, T] \rightarrow \mathbb{R}$ be a solution to $(3.5)$, satisfying $\left(u(0), u^{\prime}(0)\right) \in$ $\Upsilon_{s}$. In view of $(3.4), \mathcal{N}\left(x(0), x^{\prime}(0)\right)=\widetilde{R}_{s}$, so that $\left(x(t), x^{\prime}(t)\right) \notin \mathcal{K}_{s}$ for every $t \in[0, T]$. By Proposition 2.2, we then have that

$$
\operatorname{Rot}\left(\left(x(t)-\left(\lambda \hat{x}_{s}(t)+(1-\lambda) \hat{x}_{s}(0)\right), x^{\prime}(t)-\left(\lambda \hat{x}_{s}^{\prime}(t)+(1-\lambda) \hat{x}_{s}^{\prime}(0)\right)\right) ;[0, T]\right)
$$

is independent of $\lambda \in[0,1]$. Hence, recalling (3.4) and Claim 2,

$$
\begin{aligned}
m & <\operatorname{Rot}\left(\left(x(t)-\hat{x}_{s}(0), x^{\prime}(t)-\hat{x}_{s}^{\prime}(0)\right) ;[0, T]\right) \\
& =\operatorname{Rot}\left(\left(x(t)-\hat{x}_{s}(t), x^{\prime}(t)-\hat{x}_{s}^{\prime}(t)\right) ;[0, T]\right) \\
& =\operatorname{Rot}\left(\left(s u(t), s u^{\prime}(t)\right) ;[0, T]\right) \\
& =\operatorname{Rot}\left(\left(u(t), u^{\prime}(t)\right) ;[0, T]\right) .
\end{aligned}
$$

The lemma is then proved.

We now collect the results proved in the previous lemmas to prove Theorem 1.1 . Choose $s^{*}=s_{2}$ and fix $s \geq s^{*}$. A first solution to (1.4) is provided by Lemma 3.2. Moreover, setting $\Gamma_{0}=\partial \overline{\mathcal{B}}(0, \widetilde{r})$ and $\Gamma_{\infty}=\Upsilon_{s}$, Lemma 3.3 and Lemma 3.4 imply, via Theorem 2.3, the existence of $m+1$ (if $m$ is odd) or $m$ (if $m$ is even) nontrivial solutions to

$$
\left\{\begin{array}{l}
u^{\prime \prime}+h_{s}(t, u)=0 \\
u(0)=u(T), u^{\prime}(0)=u^{\prime}(T)
\end{array}\right.
$$

Coming back to the original equation, in view of (3.4), the proof is thus concluded.

\section{Final remarks}

In this section, we present a possible generalization of Theorem 1.1. The proof just exploits the same arguments as before.

In the following, for $a>0$, with the symbol $\lfloor a\rfloor$ we will mean the greatest integer less than or equal to $a$, while by $\lceil a\rceil$ we will denote the least integer greater than or equal to $a$. Moreover, we introduce the notation

$$
\mathcal{E}^{-}(a)=\left\{\begin{array}{ll}
\lfloor a\rfloor & \text { if } a \notin \mathbb{N}, \\
a-1 & \text { if } a \in \mathbb{N},
\end{array} \quad \mathcal{E}^{+}(a)= \begin{cases}\lceil a\rceil & \text { if } a \notin \mathbb{N}, \\
a+1 & \text { if } a \in \mathbb{N}\end{cases}\right.
$$

so that $\mathcal{E}^{-}(a) \leq\lfloor a\rfloor \leq a \leq\lceil a\rceil \leq \mathcal{E}^{+}(a)$. For instance,

$$
3=\mathcal{E}^{-}(\pi)=\lfloor\pi\rfloor<\pi<\lceil\pi\rceil=\mathcal{E}^{+}(\pi)=4,
$$

while

$$
2=\mathcal{E}^{-}(3)<\lfloor 3\rfloor=3=\lceil 3\rceil<\mathcal{E}^{+}(3)=4 .
$$


Theorem 4.1. Let $g:[0, T] \times \mathbb{R} \rightarrow \mathbb{R}$ be an $L^{\infty}$-Carathéodory function, locally Lipschitz continuous in its second variable. Assume further that:

- there exist $\delta>0$ and a continuous function $f:] 0, \delta] \rightarrow \mathbb{R}$ such that

$$
g(t, x) \leq f(x), \quad \text { for a.e. } t \in[0, T] \text {, and every } x \in] 0, \delta],
$$

and

$$
\lim _{x \rightarrow 0^{+}} f(x)=-\infty, \quad \int_{0}^{\delta} f(x) d x=-\infty,
$$

- there exists $a \in L^{\infty}(0, T)$ such that

- uniformly for almost every $t \in[0, T]$,

$$
\lim _{x \rightarrow+\infty} \frac{g(t, x)}{x}=a(t)
$$

- there exist two strictly positive constants $a_{-}, a_{+}$such that

$$
a_{-} \leq a(t) \leq a_{+}
$$

for almost every $t \in[0, T]$;

- the problem

$$
\left\{\begin{array}{l}
x^{\prime \prime}+a(t) x=w(t) \\
x(0)=x(T), x^{\prime}(0)=x^{\prime}(T),
\end{array}\right.
$$

has a unique solution $\hat{x}(t)$, and $\hat{x}(t)>0$ for every $t \in[0, T]$.

Then, there exists $s^{*}>0$ such that, for every $s \geq s^{*}$, problem (1.4) has at least

$$
2\left(\mathcal{E}^{-}\left(\frac{T \sqrt{a_{-}}}{\pi}\right)-\mathcal{E}^{+}\left(\frac{T \sqrt{a_{+}}}{2 \pi}\right)\right)+3
$$

solutions.

The expression in (4.1) highlights the fact that the elements of both $\Sigma^{D}$ and the spectrum of the $T$-periodic problem, namely

$$
\Sigma^{P}=\left\{\left(\frac{2 k \pi}{T}\right)^{2} \mid k=0,1,2, \ldots\right\},
$$

act as natural comparison quantities in the estimates of the rotation numbers of "small" and "large" solutions (around $\hat{x}_{s}(t)$ ), respectively. When 1.6) is fulfilled, i.e.

$$
\left(\frac{m \pi}{T}\right)^{2}<a_{-} \leq a_{+}<\left(\frac{(m+1) \pi}{T}\right)^{2}
$$

so that $a(t)$ is far away from both the spectra $\Sigma^{D}$ and $\Sigma^{P}$, it turns out that

$$
\mathcal{E}^{-}\left(\frac{T \sqrt{a_{-}}}{\pi}\right)=\left\lfloor\frac{T \sqrt{a_{-}}}{\pi}\right\rfloor, \quad \mathcal{E}^{+}\left(\frac{T \sqrt{a_{+}}}{2 \pi}\right)=\left\lceil\frac{T \sqrt{a_{+}}}{2 \pi}\right\rceil,
$$

and Theorem 4.1 simply reduces to Theorem 1.1 . 
The possible interest of Theorem 4.1 lies in the fact that $a(t)$ is allowed to interact with both $\Sigma^{D}$ and $\Sigma^{P}$. Indeed, as it is quite common when trying to apply the Poincaré-Birkhoff theorem, the estimates of the rotation numbers can be performed independently of any nonresonance condition, up to "correcting" the number of solutions produced (when $a(t)$ interacts with some eigenvalues). In the statement of Theorem 4.1, such a correction is made effective by means of the functions $\mathcal{E}^{-}, \mathcal{E}^{+}$. In particular, concerning the interaction with $\Sigma^{D}$, no assumptions at all are made. On the other hand, with respect to the $T$-periodic problem, we are implicitly assuming that

$$
\left\{\begin{array}{l}
x^{\prime \prime}+a(t) x=0 \\
x(0)=x(T), x^{\prime}(0)=x^{\prime}(T), \quad \Longrightarrow \quad x(t) \equiv 0 .
\end{array}\right.
$$

This property is only needed to find the first solution $\hat{x}_{s}(t)$ via topological degree arguments (see Lemma 3.2 and can hold true even in some cases when $a(t)$ jumps an arbitrarily large number of $T$-periodic eigenvalues.

As a consequence of Theorem 4.1, we observe that Corollary 1.1 holds also when $\beta \in \Sigma^{D}$, provided that $\beta \notin \Sigma^{P}$. In this case, $N_{\beta}$ will be replaced by the corrected number of solutions

$$
2\left(\frac{T \sqrt{\beta}}{\pi}-\left\lfloor\frac{T \sqrt{\beta}}{2 \pi}\right\rfloor\right)-1 .
$$

\section{References}

[1] D. Bonheure, C. Fabry and D. Smets, Periodic solutions of forced isochronous oscillators at resonance, Discrete Contin. Dyn. Syst. 8 (2002), 907-930.

[2] M. del Pino, R. Manásevich and A. Montero, T-periodic solutions for some second order differential equations with singularities, Proc. Roy. Soc. Edinburgh 120A (1992), 231-243.

[3] M. del Pino, R. Manásevich and A. Murua, On the number of $2 \pi$-periodic solutions for $u^{\prime \prime}+g(u)=s(1+h(t))$ using the Poincaré-Birkhoff Theorem, J. Differential Equations 95 (1992), 240-258.

[4] W. Y. Ding, A generalization of the Poincaré-Birkhoff theorem, Proc. Amer. Math. Soc. 88 (1983), 341-346.

[5] M. P. do Carmo, Differential Forms and Applications, Springer-Verlag, Berlin, 1994.

[6] C. Fabry and P. Habets, Periodic solutions of second order differential equations with superlinear asymmetric nonlinearities, Arch. Math. 60 (1993), 266276. 
[7] A. Fonda and L. Ghirardelli, Multiple periodic solutions of scalar second order differential equations, Nonlinear Anal. 72 (2010), 4005-4015.

[8] A. Fonda and L. Ghirardelli, Multiple periodic solutions of Hamiltonian systems in the plane, Topol. Methods Nonlinear Anal. 36 (2010), 27-38.

[9] A. Fonda, R. Manásevich and F. Zanolin, Subharmonic solutions for some second-order differential equations with singularities, SIAM J. Math. Anal. 24 (1993), 1294-1311.

[10] A. Fonda, M. Sabatini and F. Zanolin, Periodic solutions of perturbed Hamiltonian systems in the plane by the use of the Poincaré-Birkhoff Theorem, preprint, 2011.

[11] A. Fonda and R. Toader, Radially symmetric systems with a singularity and asymptotically linear growth, Nonlinear Anal. 74 (2011), 2485-2496.

[12] J. K. Hale, Ordinary Differential Equations, Krieger, New York, 1980.

[13] A. Lazer and P. J. McKenna, Large scale oscillatory behaviour in loaded asymmetric systems, Ann. Inst. H. Poincaré Anal. Non Linéaire 4 (1987), 243-274.

[14] P. Le Calvez and J. Wang, Some remarks on the Poincaré-Birkhoff theorem, Proc. Amer. Math. Soc. 138 (2010), 703-715.

[15] C. Rebelo, Periodic solutions of nonautonomous planar systems via the Poincaré-Birkhoff fixed point theorem, Ph. D. Thesis, Lisbon, 1996.

[16] C. Rebelo, A note on the Poincaré-Birkhoff fixed point theorem and periodic solutions of planar systems, Nonlinear Anal. 29 (1997), 291-311.

[17] C. Rebelo, Multiple periodic solutions of second order equations with asymmetric nonlinearities, Discrete Contin. Dynam. Systems 3 (1997), 25-34.

[18] C. Rebelo and F. Zanolin, Multiplicity results for periodic solutions of second order ODEs with asymmetric nonlinearities, Trans. Amer. Math. Soc. 348 (1996), 2349-2389.

[19] C. Rebelo and F. Zanolin, Multiple periodic solutions for a second order equation with one-sided superlinear growth, Dynam. Contin. Discrete Impuls. Systems 2 (1996), 1-27.

[20] P. J. Torres, Existence of one-signed periodic solutions of some second-order differential equations via a Krasnoselskii fixed point theorem, J. Differential Equations 190 (2003), 643-662. 
[21] C. Zanini and F. Zanolin, A multiplicity result of periodic solutions for parameter dependent asymmetric non-autonomous equations, Dyn. Contin. Discrete Impuls. Syst. Ser. A Math. Anal. 12 (2005), 343-361.

Authors' addresses:

Alberto Boscaggin and Maurizio Garrione

SISSA - International School for Advanced Studies

Via Bonomea 265

I-34136 Trieste

Italy

e-mail: boscaggi@sissa.it, garrione@sissa.it

Alessandro Fonda

Dipartimento di Matematica e Informatica

Università di Trieste

P.le Europa 1

I-34127 Trieste

Italy

e-mail: a.fonda@units.it 